\title{
A novel scalable electrode array and system for non-invasively assessing gastric function using flexible electronics
}

\section{Armen Gharibans}

University of Auckland

\section{Tommy Hayes}

University of Auckland

Daniel Carson

University of Auckland

\section{Stefan Calder}

Alimetry

\section{Chris Varghese}

University of Auckland https://orcid.org/0000-0001-7369-8639

\section{Peng Du}

University of Auckland, Auckland Bioengineering Institute

\section{Yaara Yarmut}

Alimetry

\section{Stephen Waite}

Alimetry

\section{Celia Keane}

University of Auckland

Jon Woodhead

Alimetry

\section{Christopher Andrews}

Alimetry

Gregory 0'Grady ( $\square$ greg.ogrady@auckland.ac.nz )

University of Auckland

\section{Article}

Keywords: Bioelectronics, functional gastrointestinal disorders, gastric motility, diagnostics

Posted Date: January 12th, 2022

DOl: https://doi.org/10.21203/rs.3.rs-1233270/v1 
License: (c) (i) This work is licensed under a Creative Commons Attribution 4.0 International License. Read Full License 


\section{Abstract}

Disorders of gastric function are highly prevalent, but diagnosis often remains symptom-based and inconclusive. Body surface gastric mapping is an emerging diagnostic solution, but current approaches lack scalability and are cumbersome and clinically impractical. We present a novel scalable system for non-invasively mapping gastric electrophysiology in high-resolution (HR) at the body-surface. The system comprises a custom-designed flexible HR sensor array and portable data-logger synchronized to an App, with automated analysis and visualization algorithms. The novel system underwent performance testing then validation in 24 healthy subjects. In all subjects, gastric electrophysiology and meal responses were successfully captured and mapped non-invasively (mean frequency $2.9 \pm 0.3$ cycles per minute; peak amplitude at mean $60 \mathrm{~m}$ postprandially with return to baseline in $<4 \mathrm{~h}$ ). Spatiotemporal mapping showed regular and consistent wave activity of mean direction $182.7^{\circ} \pm 73(74.7 \%$ antegrade, $7.8 \%$ retrograde, $17.5 \%$ indeterminate). The presented system is a new diagnostic tool for assessing gastric function that is scalable, validated, and ready for clinical applications, offering several biomarkers that are new to gastroenterology practice.

\section{Introduction}

Chronic gastric symptoms affect up to $10 \%$ of adults, are increasing in prevalence, and impart a vast quality of life and cost burden. 1,2,3,4 These symptoms encompass early satiation, excessive postprandial fullness, epigastric pain and burning, nausea and vomiting, and are clinically recognised in functional dyspepsia, chronic nausea and vomiting syndromes, and gastroparesis. ${ }^{5}$ However, accurately defining, differentiating and subtyping these overlapping conditions remains a critical problem in gastroenterology, owing to a lack of objective diagnostic tests. The only widely-available test of gastric function is measurement of gastric emptying, which is used to define gastroparesis, but is controversial due to its limited specificity, weak correlation with symptoms, and inconsistency over time. ${ }^{4,6}$ There is a need for new tests of gastric function, and an ideal test would offer actionable biomarkers while being safe, noninvasive, scalable and accessible, and cost-efficient. ${ }^{7}$

A century ago, Walter C. Alvarez introduced electrogastrography (EGG) as a non-invasive diagnostic tool for gastric function. ${ }^{8}$ EGG measures the bioelectrical slow waves that coordinate gastric motility, and also registers gastric contractile activity through an increase in signal power. ${ }^{9,10} \mathrm{~A}$ substantial literature has been generated to show that EGG abnormalities are consistently prevalent in patients with gastric symptoms, ${ }^{11-13}$ yet EGG failed to achieve common clinical adoption. Limitations to EGG include its focus on frequency as the predominant measure of abnormality, sensitivity to noise that could lead to misinterpretation of data, and an inability to account for a wide variability in gastric anatomical position. ${ }^{14,15}$

Recently, Body-Surface Gastric Mapping (BSGM; high-resolution EGG) has been proposed as a novel diagnostic method to overcome the limitations of EGG, by employing a dense grid of electrodes to 
measure and map gastric activity in high-resolution at the epigastrium. ${ }^{14,16,17}$ The convergence of an improved physiological understanding of gastric slow wave patterns in health vs disease states, ${ }^{18}$ modern amplifiers, ${ }^{19}$ and filters that more robustly discriminate gastric activity from noise, ${ }^{20,21}$ have revitalized interest in the clinical potential of gastric electrophysiology. ${ }^{14}$ Recent studies have shown that BSGM can identify novel biomarkers such as slow wave direction or pattern that offer superior symptom correlations compared to traditional EGG and gastric emptying testing. ${ }^{16,17}$

Until now, the use of body surface mapping has been restricted to research applications. This is because the test has been impractical, involving the time-consuming placement of many individual electrodes and managing the associated cable bundle, which is also a cleaning and disinfection barrier to clinical use. In addition, BSGM has required expensive specialized acquisition systems restricted to investigational use, and complex analysis approaches requiring substantial signal processing expertise. In this work, we developed a novel non-invasive clinical solution for BSGM that overcomes these problems through the application of flexible electronics and miniaturized wearable circuitry, together with advances in data processing and visualization. The novel BSGM system and method was subjected to extensive benchtop verification, followed by an experimental evaluation to demonstrate its reliability in generating new and improved clinical biomarkers of human gastric function, including robust frequency and power spectra, the direction and pattern of wave travel, and meal response metrics.

\section{Materials And Methods}

\section{System Overview}

The novel BSGM system was designed to accommodate a clinical testing framework comprising a 30minute fasting baseline, consumption of a standardized meal, and up to 4 hours of postprandial testing (Fig. 1). This timeframe was chosen to reflect the typical period of the gastric meal response. ${ }^{22}$ The BSGM system (overviewed in Fig. 2) is comprised of a flexible and conformable pre-gelled 'peel-and-stick' high-resolution (HR) sensor array, an ambulatory data logger with custom electronics specifically tuned for gastric bioelectrical data, and a native iOS App with HIPAA-compliant cloud connectivity and Bluetooth 5.0 synchronization to the data logger. The App guides the user through test setup, including user-specific algorithm guided array positioning, requests symptom data from the subject during testing according to a validated design, and manages data transfers. Automated algorithms were also developed for filtering and extracting and visualizing clinical biomarkers. Each of these components are discussed in further detail below.

\section{Sensor Array}

Gastric bioelectrical signals are of weak amplitude and signal strength diminishes exponentially as distance from the source increases, ${ }^{23}$ meaning that electrodes should be positioned directly over the stomach for capturing reliable data. ${ }^{24}$ An electrode sensor array was therefore designed to a size that 
was capable of achieving a position consistently overlying the stomach with high reliability when placed on the epigastrium (196 $\mathrm{cm}^{2}$; data based on a separate proprietary anatomical study). The sensor array (Fig. 2A, 3A) was screen printed in layers on a $21 \times 16 \mathrm{~cm}$ thermoplastic polyurethane (TPU) substrate. TPU was chosen for its ease of manufacturing, biocompatibility, and high conformability, allowing comfortable adherence to the user's epigastrium (Fig. 3B), including through a full range of movement without delaminating from the skin.

An $8 \times 8$ grid of electrodes ( $11 \mathrm{~mm}$ diameter, $20 \mathrm{~mm}$ center-to-center electrode spacing) with adjacent reference and ground electrodes was screen-printed onto the TPU substrate using $\mathrm{Ag} / \mathrm{AgCl}$ ink. Each electrode pad has an associated conductive track coalescing to a 'mating panel' (Fig. 2B). An insulating dielectric layer was then applied to coat the entire surface except for the electrode pads and mating panel (indicated by blue areas in Fig 2B). A conversion process was then undertaken (Fig. 3A). First, an adhesive laminate was applied over the dielectric layer. Conductive hydrogel discs were then positioned on each $\mathrm{Ag} / \mathrm{AgCl}$ electrode to ensure low-impedance charge transfer at the skin interface. ${ }^{25}$ Finally, the adhesive and hydrogel layers were covered by a backing layer, which is peeled off immediately prior to application. The entire sensor array was mass-fabricated with an automated process involving roll-to-roll screen-printing, die-cutting, and converting machinery, before individual packaging in moisture barrier foil pouches (Alimetry Ltd, New Zealand).

A total of 22 completed arrays underwent comprehensive testing to ensure adequate electrode quality and performance according to the ANSI/AAMI EC12:2000 Standard and a further 30 arrays underwent shelf-life testing. Further details regarding the array testing procedure can be found in the Supplementary

\section{Methods.}

\section{Connector}

A compact board-to-board connector design was introduced that overcomes a key design challenge in the field of high-resolution wearable electronics, being the attachment of a flexible array to a rigid circuit without the need for bulky connector attachments or cables. The sensor array interfaces with the data logger device by means of a custom flexible printed circuit cable and high-density compression connector with a $0.2 \mathrm{~mm}$ thick PI stiffener on the back of the connector end, placed between a machined stainlesssteel clamp (Alimetry Ltd, New Zealand) (Fig. 2C). The other end of the cable attaches to a zero-insertionforce connector on a custom mating PCB that allows repeatable connections to the data logger without additional tools.

\section{Data Logger}

An ambulatory data logger (Fig. 2A) was designed and fabricated, employing all custom electronics and firmware, and housed within an off-the-shelf casing (Hammond Manufacturing, USA; 147 x 89 x 25 mm). Bioelectrical signals are recorded at $250 \mathrm{~Hz}$, amplified, and digitized by low-noise programmable gain 
amplifiers with each input compared against a common reference electrode as shown on Fig. 2A, to provide unipolar recordings for 64 channels. Data is stored on removable internal storage until uploaded to a HIPAA-compliant cloud server via the App. An onboard accelerometer is used to record motion during the recording. Bluetooth connection with the App is maintained throughout the recording session, and to facilitate data upload. After a successful upload, data is securely deleted from the data logger in preparation for the subsequent test. After assembly, the data logger electronics underwent comprehensive electrical performance testing to ensure design criteria were met, as well as electromagnetic compatibility (EMC) testing. Further details regarding these testing methods are provided in the Supplementary

\section{Methods.}

\section{App and Array Placement Algorithm}

The companion App was programmed in Swift v.5.1, being designed for use on an iPad mini (Apple, CA, USA). A password-protected administration section allows the user to register the test and participant details, customize recording variables, and to guide setup. As noted above, reliable placement of the electrode array directly over the stomach location is an essential design requirement for reliable data capture in body surface gastric mapping. The App therefore further incorporated an array positioning algorithm taking into account guided measurements between xiphoid and umbilicus, xiphoid and anterior

superior iliac spine (ASIS), and abdominal circumference (Fig. 2Di). ${ }^{26}$ These measurements were used to calculate a patient-specific array location with reference to the umbilicus, which is displayed to the user. Guided placement of the array by this algorithm, in conjunction with the chosen array size, aimed to reliably capture the gastric field within the recording electrode in the high majority of participants, by accounting for known anatomical variations (verified in a previous proprietary anatomical study). ${ }^{26}$ The App also undertakes an impedance check of the array prior to test initiation to ensure optimal data quality (Fig. 2Dii).

\section{Clinical and Analytical Methods}

Ethical approval for the clinical studies was obtained from the Auckland Health Research Ethics Committee (AHREC, reference AH1130). This study focused on clinical evaluation of the novel system in 24 healthy subjects to demonstrate reliability for outputting each of the stated gastric biomarkers. Healthy subjects were 18 years or older with no known active GI symptoms or pathology, not meeting Rome IV criteria for a functional GI disorder, and not taking any medication known to affect gastrointestinal motility including anxiolytics and antidepressants. Additional exclusion criteria were any of the following: metabolic, neurogenic, or endocrine disorder known to cause gastric dysmotility (including scleroderma, multiple sclerosis and hyperthyroidism), active GI infection, inflammatory bowel disease, previous gastric or esophageal surgery, history of GI malignancy, open abdominal wounds or abdominal skin not intact, fragile skin, allergy to adhesives and pregnancy. Additionally, those with body mass index $(\mathrm{BMI})>35$ were excluded, as data reliability is currently uncertain above this threshold. ${ }^{24}$ 


\section{Experimental protocol}

Subjects were fasted for at least eight hours and were asked to not use nicotine or consume caffeine in the morning of their study. Measures of chronic gut symptoms were evaluated by the validated Patient Assessment of Gastrointestinal Disorders Symptom Severity Index (PAGI-SYM) ${ }^{27}$ and Patient Assessment of Upper Gastrointestinal Disorders-Quality of Life (PAGI-QOL) ${ }^{28}$ questionnaires. The anterior abdomen was shaved if required and the skin prepped using a conductive gel (NuPrep; Weaver and Company, CO, USA). The novel flexible sensor array was then placed on the epigastrium, guided by the positioning algorithm. As per Fig. 1, gastric activity was measured over a 30-minute fasted period, followed by consumption of a standardized meal over 10 minutes, including a $232 \mathrm{kcal}$ nutrient drink (230 mL Ensure; Abbott Nutrition, IL, USA) and an oatmeal energy bar (250 kcal with $5 \mathrm{~g}$ fat, $45 \mathrm{~g}$ carbohydrate, $10 \mathrm{~g}$ protein, $7 \mathrm{~g}$ fiber; Clif Bar \& Company, CA, USA), and a further 4 hour postprandial recording. Subjects remained reclined at 45 degrees for the entirety of the recording duration with their legs elevated in a comfortable position, and were instructed to limit movement and talking, avoid sleeping, and refrain from touching the array. ${ }^{29}$ At the end of the test, any adverse events were recorded and subjects were given a short questionnaire regarding system usability. Comfort during the test and any discomfort on removal of the array were assessed using electronic 100-point visual analogue scales ( 0 - "very uncomfortable" to 100 "very comfortable" for the test; and 0 "not painful" to 100 "most painful imaginable" for the array removal).

\section{Signal Processing and Analysis}

Data collected using the BSGM device were processed using an automated proprietary algorithm that enabled filtering, biomarker outputs, and visualizations. In brief, each of the 64 channels were analyzed to first remove segments of significant artifact based on the methods of Gharibans et al. ${ }^{21}$ Further steps in the algorithm then generated the biomarkers of gastric function. Spatial heat maps were generated to show the predicted gastric location within the mapped field according to a power spectrum. Spectral analyses were performed using a composite of channels located centrally over the gastric position in the heat map, by a short-time Fourier transform (4 minute windows with $75 \%$ overlap), visualized as frequency-amplitude and amplitude-time plots. ${ }^{21}$ Dominant frequency (cycles per minute; cpm), mean amplitude $(\mu \mathrm{V})$, and variance in the dominant frequency were calculated for each participant and as summary statistics for the whole cohort. Meal response was characterized by the increase in the power of the spectral analysis after a meal (power ratio) and was calculated separately for the first 2 hours postprandially $\left(\mathrm{PR}_{2 \mathrm{~h}}\right)$ and the entire postprandial period $\left(\mathrm{PR}_{4 \mathrm{~h}}\right)^{30}$. Average dominant frequency was calculated for the $\mathrm{PR}_{2 \mathrm{~h}}$ phase when signal power is high. The duration taken to return to a stable baseline was also calculated in each period with reference to the fasting period. The frequency-amplitude spectrograms were also averaged, after normalizing amplitude for each participant, to define overall trends in the meal response power curve across the cohort. Mean amplitude was correlated against BMI. 
Spatiotemporal metrics were derived for each subject using methods similar to those described by

Gharibans et al. ${ }^{16,20}$ Wave patterns were visualized as propagation animations, ${ }^{23}$ and their directionality was defined by manual classification. This was performed by 5 independent reviewers with conflicts resolved by consensus panel. Each reviewer visually assessed the animated data in $15 \mathrm{~m}$ epochs and classified these as antegrade, retrograde, indeterminate, or low-amplitude noise, with the latter being excluded from subsequent percentage calculations. Summary data on wave directions were also computed via the algorithm and displayed as polar histograms. ${ }^{16,20}$

\section{Statistical analysis}

Normality was assessed by visual inspection of Q-Q plots. Continuous independent normal variables were compared using Student's t-test, and continuous independent non-normal variables using the MannWhitney $\mathrm{U}$ test. More than two sets of continuous dependent normal variables were compared using the repeated measures ANOVA, with a Bonferroni post-test correction applied. More than two sets of continuous dependent non-normal variables were compared using Friedman's test, with a Dunn correction for multiple comparisons applied. Strength of association between variables was determined using Pearson's rank correlation coefficient ( $r$ ). Sample size calculations for Array testing can be found in the Supplementary Methods. The statistical significance threshold was $p<0.05$. All statistical analysis was performed using GraphPad Prism v.8 (GraphPad Software, CA, USA) and R version 4.0.3 (R Foundation for Statistical Computing, Vienna, Austria). Values are reported as the mean \pm standard deviation (SD) unless stated otherwise.

\section{Results}

\section{Flexible array testing}

The mass-fabricated flexible arrays were tested to ensure the maximum Euclidean distance between ink layers was at most $1 \mathrm{~mm}$, each hydrogel pad was only in contact with a singular $\mathrm{Ag} / \mathrm{AgCl}$ electrode and that no adhesive layer overlapped with hydrogel pads. Based on testing of 22 arrays as determined by the a priori sample size calculation, all distances between $\mathrm{Ag} / \mathrm{AgCl}$ and $\mathrm{Ag}$ layers for all electrodes were less than $1 \mathrm{~mm}$; all distances between $\mathrm{Ag} / \mathrm{AgCl}$ and dielectric layers for all electrodes were less than $1 \mathrm{~mm}$; each hydrogel pad was in contact with exactly $1 \mathrm{Ag} / \mathrm{AgCl}$ electrode for all the electrodes on all the arrays and the adhesive layer did not overlap the hydrogel layer on any electrodes.

The array electrical testing met ANSI/AAMI EC12:2000 ECG Electrode Standards. Accordingly, electrical testing of a random pair of electrodes across 22 arrays demonstrated that the average impedance across all 22 electrode pairs was $0.467 \mathrm{k} \Omega$ (none exceeding $3 \mathrm{k} \Omega$ ), maximum DC voltage offset was less than $100 \mathrm{mV}$ throughout 8 hours of testing (maximum $15.2 \mathrm{mV}$ ), and maximum peak-to-peak (p-p) noise was less than $150 \mu \mathrm{V}$ throughout random 5-minute periods at a random electrode pair from 22 arrays (maximum $22.8 \mu \mathrm{V}$ ). The resistance between all neighboring electrodes on all arrays was greater than 1 
$\mathrm{M} \Omega$, indicating sufficient electrode isolation to assess gastric propagation patterns. Array shelf-life testing was performed on 30 additional arrays tested at both accelerated 1 and 2 year periods, confirming at least a 2 year shelf-life. The array therefore met the required standards of production quality.

\section{Data logger testing}

Electrical performance testing of the data logger demonstrated that internal noise referred to inputs did not exceed $1 \mu \mathrm{V} p$-v when all inputs were connected together and the amplitude response between the range $0.01 \mathrm{~Hz}$ to $0.25 \mathrm{~Hz}$ was between $99.3 \%-100 \%$ relative to the amplitude response at $0.05 \mathrm{~Hz}$ across 3 data loggers (Fig. S1). The amplitude range measured by all 64 channels for a $1000 \mu \mathrm{Vp}-\mathrm{p} 0.05 \mathrm{~Hz}$ test input signal was between $1000.8 \mu \mathrm{V}$ and $1004.4 \mu \mathrm{V}$. The channel-to-channel skew was 0 ms between all channels; all results were within the $\pm 1 \%$ acceptable range (i.e., between $990 \mu \mathrm{V}$ to $1010 \mu \mathrm{V}$ ). The average amplitude for all channels throughout the entire 5-hour test was 1001.2 $\mu \mathrm{Vp}-\mathrm{p}, 1001.7 \mu \mathrm{Vp}-\mathrm{p}$, and $1001.6 \mu \mathrm{Vp}$-p for each Data Logger respectively. The frequency for all channels was $0.0500 \mathrm{~Hz}$ for each channel and maintained throughout the 5 hours. The resistance between every possible combination of inputs on all 3 Data Loggers was greater than $1 \mathrm{M} \Omega$.

\section{Clinical Evaluation}

Twenty-four healthy subjects ( 16 male; 8 female) participated, of median age 28.5 (range 20-73 years). The mean BMI was $24.2 \pm 3.5 \mathrm{~kg} / \mathrm{m}^{2}$ (range $17.9-31.2$ ), with 15 subjects of $\mathrm{BMI}<25$, and 9 of $\mathrm{BMI} \geq 25$ ). These healthy control subjects reported a minimal GI symptom burden (mean PAGI-SYM $0.17 \pm 0.33$; mean GCSI $0.21 \pm 0.40$ ) and a high GI-related quality of life (mean total PAGI-QOL $4.84 \pm 0.26$ ).

\section{Stomach localization, impedance, and artifact}

The sensor array positioning algorithm captured the area of high signal power from the stomach accurately in all 24 subjects. In 22/24 (92\%) participants this area was located centrally within the mapped field, vs nearer to the edge of the mapped field in the remaining 2/24 (8\%) (Fig. 4A). The average heat map from all 24 recordings is shown in Fig. 4B, confirming an overall average central position. The mean impedance was $84.1 \mathrm{k} \Omega$ (SEM $10.8 \mathrm{k} \Omega$ ) (Fig. 4C). The high majority of data were usable for analysis following automated removal of contamination by artifacts and subsequent signal recovery (mean $94.8 \pm 4.8 \%$ ).

\section{Frequency, Amplitude, and Meal Response}

Clear dominant frequency bands were identified in all patients (e.g., Fig. 5A,B). Mean dominant frequency was $2.9 \mathrm{cpm} \pm$ s.d. 0.3 (range 2.4 to $3.4 \mathrm{cpm}$ ), which was stable within participants (mean intra-subject variability $\pm 0.43 \mathrm{cpm}$, range $0.10-0.83$ ). Mean amplitude over the $4.5 \mathrm{hr}$ test duration ranged from 17.1 to 
$65.3 \mu \mathrm{V}$ across the cohort (mean $35.2 \pm$ SEM 2.7). The mean fasted amplitude was $24.2 \pm$ SEM $2.2 \mu \mathrm{V}$, which was stable within participants (mean intra-subject variability $\pm 3.7 \mu \mathrm{V}$, range 0.6-7.2). Mean amplitude significantly increased during the 0 -2-hour post-prandial period (mean $39.1 \mu \mathrm{V} \pm$ SEM 3.2; $p<0.001$ vs fasted period) and during the $2-4$ hour post-prandial period was mean $34.3 \mu \mathrm{V} \pm$ SEM 3.2 $\left(p=0.0001\right.$ vs fasted period) (Fig. 6A). Power ratios were: $P_{2 h} 1.75 \pm$ s.d. 0.90 and $P R_{4 h} 1.61 \pm$ s.d 0.73 . Amplitude was negatively correlated with BMI ( $r=-0.41 ; p=0.046$; Fig. 6B).

Three examples of meal responses are shown with frequency-amplitude spectrograms in Fig. 5A. These cases demonstrate the variability present in the time to maximum post-prandial amplitude, which could occur nearly immediately after the meal (e.g., Fig. 5Ai, ii), or following a lag-phase (e.g., Fig. 5Aiii). Fig. $5 \mathrm{~A}$ also demonstrates the variability in the length of the meal response (i.e., duration before return to baseline). The post-prandial amplitude returned to baseline by four hours post-meal in 21/24 subjects; the remaining subjects either did not show a substantial increase in power above baseline $\left(n=2 ; P R_{2 h} 1.03\right.$ and 1.23), or amplitude did not return to baseline prior to the end of the test $\left(n=1 ; P R_{2 h} 1.21\right.$ vs $P_{3}$ $4 \mathrm{~h}$ 2.72). The average frequency-amplitude plot for all subjects is presented in Fig. 5B. This data shows that, on-average, amplitude steadily increased and peaked within $2 \mathrm{~h}$ after the meal, before gradually returning towards baseline fasted values by the end of the test.

\section{Wave Propagation Profiling}

Spatiotemporal wave propagation profiling was performed in all 24 subjects. Example data are shown in Fig. 7, with Fig. 7A, i and Fig. 7B, ii showing examples of typical antegrade propagation (see also Supplementary Animations). On classification, $74.7 \%$ of all subjects recordings were antegrade, $7.8 \%$ retrograde, and $17.5 \%$ were indeterminate due to apparent looping of the body surface dipole that precluded clear determination of direction. ${ }^{31}$ An example of retrograde activity is shown in Fig. 7 B, i. Retrograde waves were observed in 10/24 subjects, typically during the first 1.5 hours postprandially, lasting median 22.5 minutes, and being sustained for $>15$ minutes in 7 subjects. Mean wave direction in the first $2 \mathrm{~h}$ postprandially was $182.7^{\circ} \pm 37$ (Fig. 7C). The average distribution of wave direction across all subjects is shown in the phase map in Fig. 7D, demonstrating dominant antegrade propagation.

\section{Safety}

No participants had significant adverse events. One participant reported low-grade itchiness related to the sensor array (resolution within 30 minutes), and another had moderate erythema of the epigastrium upon removal. Discomfort on removal of the sensor array was low for most subjects (mean $26.5 \pm 22.2$ / 100), while comfort during the test was high (mean $69.9 \pm 27.1$ / 100).

\section{Discussion}


In this study we present a novel scalable flexible electrode array and complete clinical system for bodysurface gastric mapping (BSGM). To our knowledge, the flexible electronics array comprises the first mass-produced peel-and-stick solution for body surface mapping applications. First human data confirm that a dense field of gastric signal data was effectively captured in all subjects, enabling several biomarkers of gastric activity to be accurately characterized, including frequency and power spectra, meal response characteristics, and spatiotemporal metrics of wave propagation. These data demonstrate that the new system is a reliable non-invasive tool for characterizing gastric electrophysiology at highresolution, offering a translational platform that is now ready for clinical applications. The test was well tolerated by all subjects, with no significant adverse events.

The BSGM system is shown to overcome several of the key technical limitations of EGG. ${ }^{14,32}$ First, the combination of the large array size and a patient-specific positioning system meant that the region of highest gastric signal power could be routinely and reliably captured within the mapped field. This is essential because amplitude falls exponentially as electrodes move off the stomach's location, ${ }^{23}$ which is highly variable. ${ }^{26,33}$ Moreover, summating multiple signals from the region of highest gastric power substantially improves the accuracy of spectral analysis, reducing false positive data. ${ }^{24}$ Second, defining gastric propagation patterns is a critical advance over EGG, because gastric dysrhythmias are now recognized as being spatially complex, with abnormal propagation patterns now known to occur at normal frequencies. ${ }^{18,34}$ Third, the novel system integrates multiple technical improvements that together enhance signal-to-noise discrimination, which was a further key pitfall with EGG interpretation. ${ }^{15}$ These improvements included modern high-fidelity bioamplifiers embedded in custom hardware and firmware specifically tuned to gastric parameters, a conformable electrode achieving low impedance at the skin interface, and an automated artifact-rejection scheme. ${ }^{21}$

The solution introduced here also represents many advances over previous BSGM systems used in research applications. Gharbians et al and Somarajan et al. employed $5 \times 5$ grids of individually-placed cardiac electrodes, ${ }^{16,20,17}$ while Bradshaw et al. employed a $4 \times 4$ grid. ${ }^{35}$ All of these systems required individual electrodes and cables, being cumbersome for clinical use and involving long setup times, as well as being difficult to reliably clean to hospital standards. Individual electrode systems are also subject to inconsistent spacing and higher risk of crosstalk. Our flexible and conformable mass-fabricated electrode array overcomes these issues while obviating the need for prior imaging of the stomach's location due to its large size combined with an individualized positioning algorithm. The electrode density is much greater than previous approaches (including those employing flexible electronics ${ }^{36}$ ), enabled by the introduction of a screen-printed solution, careful optimization of track routing, and a novel interposed clamp connector. This connector efficiently overcomes the problems of mating a stretchable array with a rigid circuit board and could therefore find utility in other fields of body-surface electronics including cardiac, neural, and skeletal muscle applications. Finally, the system also remains portable and userfriendly, including an App to guide rapid standardized setup. 
The presented system is fully scalable with arrays already being mass-fabricated under ISO13485 standards and is ready for clinical application. This is timely, because the objective assessment of gastric function is an area of current clinical concern. Gastric emptying measurement has recently fallen under renewed scrutiny after a prominent study reported that it did not adequately classify patients and was inconsistent over time. ${ }^{6}$ Gastric emptying correlates with symptoms when optimally performed, albeit weakly, ${ }^{37}$ but its role in guiding diagnosis and management remains controversial. ${ }^{38}$ Meanwhile, standard electrogastrography (EGG) is not currently recommended for clinical use, ${ }^{39}$ partly due to the limitations described above, ${ }^{14,32}$ while other tests such as antroduodenal manometry and fundal accommodation testing generally remain restricted to niche applications and/or specialist centers. ${ }^{40,41}$ New tests are needed that reference underlying pathophysiological mechanisms and provide actionable biomarkers, in order to progress from symptom-based diagnosis, diagnosis by exclusion, and trial-anderror therapy. ${ }^{7,38}$ BSGM appears promising because it includes several biomarkers that hold promise independently or cumulatively, and which correlate with symptoms in emerging datasets, ${ }^{16}$ while being non-invasive and accessible in any outpatient setting. ${ }^{38}$

The next step will be to apply the BSGM system in large cohorts of patients and controls to robustly assess clinical utility. The current data provides an indication of normal ranges for BSGM biomarkers, but it would be valuable to expand these to reference ranges, and then to evaluate whether these differ by demographics and with alternative meals. It was notable that a small proportion of gastric activity in our healthy subjects propagated in a retrograde direction (7.8\% of mapped waves). Retrograde activation was not observed on previous invasive gastric mapping studies in fasted healthy subjects, ${ }^{42,43}$ except for a single instance in an over-inflated stomach, ${ }^{34}$ but has been reported post-prandially in two other recent BSGM research studies, ${ }^{16,17}$ and could relate to gastric distension. ${ }^{44}$ It therefore appears that retrograde wave behavior is part of the normal post-prandial gastric repertoire, which would be an important physiological discovery, ${ }^{45}$ but this finding requires further scrutiny in dedicated studies before it can be accepted. Gastric electrophysiology has recently been confirmed by meta-analyses to significantly deviate from healthy subjects in FD, CNVS, gastro-esophageal reflux disease, and various pediatric disorders with traditional EGG, ${ }^{11-13,46}$ indicating priority target groups for future clinical studies using BSGM. Additionally, studies suggest that dysrhythmias may also be implicated in a subset of postoperative gastric dysfunction. ${ }^{47,48}$ Another important step will be to continue correlations of symptoms with specific biomarkers detected by BSGM, in order to further define pathophysiological features. ${ }^{49}$

This study is the first to suggest that a full meal response cycle can be robustly and routinely profiled non-invasively at the body surface. In our healthy subjects, the post-prandial power increase typically trended back to baseline by 3.5 hours or earlier, with only one subject still showing elevated power at 4 hours (Fig. 5). The hypothesis is therefore presented that this meal response duration correlates with gastric emptying time. Historically, the typically 1-hour EGG test has failed to correlate with gastric emptying, ${ }^{32}$ but this should now be re-evaluated with BSGM in dedicated head-to-head testing. Gastric 
emptying is a function of caloric density and meal volume, ${ }^{50}$ and our test meal comprises a higher nutrient intake than a standard gastric emptying test ( $480 \mathrm{kCal}$ vs $255 \mathrm{kCal})$, which usually completes before 4 hours. ${ }^{22}$ Smaller meals would therefore be warranted for gastric emptying comparisons.

Signal processing and analysis methods remain in ongoing development. A recent technical paper has validated the accuracy of BSGM in measuring the propagation direction of individual wavefronts at the body surface with direct reference to simultaneous high-resolution serosal mapping, using similar techniques to those applied here. ${ }^{51}$ This study provides confidence that the technique is reliable, while also paving the way for future studies that provide more granular data on individual wave direction throughout entire recordings, ${ }^{17,35}$ This step will also enable temporal correlations between symptom onset and retrograde wave patterns, in order to further elucidate the emerging clinical significance of retrograde gastric propagation..$^{52}$ Modeling and bench-top studies also suggest that it may be possible in future to identify more complex wave patterns that occur in the stomachs of patients (e.g. colliding wavefronts, conduction blocks and re-entrant activity), ${ }^{53}$ although this has not yet been validated experimentally. An additional limitation is that this study was performed in healthy subjects of normal weight, and the reliability of the system requires further validation in obese subjects. Previously, Gharibans et al have shown the ability to measure BSGM data up to a BMI of at least 35 , which was therefore applied as the cut-off here. ${ }^{24}$

In summary, our novel flexible electrode array and BSGM system provides a robust clinical solution for non-invasively profiling gastric electrophysiology at the body surface. The system is scalable, validated, and ready for clinical applications, offering several biomarkers that are new to gastroenterology practice.

\section{Declarations}

\section{Acknowledgements, Funding and Disclosures}

\section{Funding}

This work was supported by the New Zealand Health Research Council and the Royal Australasian College of Surgeons' John Mitchell Crouch Fellowship.

\section{Conflicts of Interest}

AG, PD, CNA, GO hold grants and intellectual property in the field of GI electrophysiology and are members of University of Auckland spin-out companies: The Insides Company (GO), FlexiMap (PD), and Alimetry (AG, SC, YY, SW, JSTW, PD, CNA and GO). DAC, TCLH, and CV have no relevant conflicts to declare. 


\section{References}

1. Aziz I, Palsson OS, Törnblom H, Sperber AD, Whitehead WE, Simrén M. Epidemiology, clinical characteristics, and associations for symptom-based Rome IV functional dyspepsia in adults in the USA, Canada, and the UK: a cross-sectional population-based study. Lancet Gastroenterol Hepatol. 2018;3(4):252-262.

2. Aziz I, Palsson OS, Whitehead WE, Sperber AD, Simrén M, Törnblom H. Epidemiology, Clinical Characteristics, and Associations for Rome IV Functional Nausea and Vomiting Disorders in Adults. Clin Gastroenterol Hepatol. 2019;17(5):878-886.

3. Lacy BE, Weiser KT, Kennedy AT, Crowell MD, Talley NJ. Functional dyspepsia: the economic impact to patients. Aliment Pharmacol Ther. 2013;38(2):170-177.

4. Andreasson A, Talley NJ, Walker MM, et al. An Increasing Incidence of Upper Gastrointestinal Disorders Over 23 Years: A Prospective Population-Based Study in Sweden. American Journal of Gastroenterology. 2021;116(1):210-213. doi:10.14309/ajg.0000000000000972

5. Stanghellini V, Chan FKL, Hasler WL, et al. Gastroduodenal Disorders. Gastroenterology. 2016;150(6):1380-1392.

6. Pasricha PJ, Grover M, Yates KP, et al. Functional Dyspepsia and Gastroparesis in Tertiary Care are Interchangeable Syndromes With Common Clinical and Pathologic Features. Gastroenterology. 2021;160(6):2006-2017.

7. Camilleri M, Chedid V. Actionable biomarkers: the key to resolving disorders of gastrointestinal function. Gut. 2020;69(10):1730-1737.

8. THE ELECTROGASTROGRAM AND WHAT IT SHOWS. JAMA: The Journal of the American Medical Association. 1922;78(15):1116. doi:10.1001/jama.1922.02640680020008

9. Smout AJ, van der Schee EJ, Grashuis JL. What is measured in electrogastrography? Dig Dis Sci. 1980;25(3):179-187.

10. Zhu H, Chen JDZ. Gastric distension alters frequency and regularity but not amplitude of the gastric slow wave. Neurogastroenterology and Motility. 2004;16(6):745-752. doi:10.1111/j.13652982.2004.00571.x

11. Bhat S, Varghese C, Carson DA, et al. Gastric dysrhythmia in gastroesophageal reflux disease: a systematic review and meta-analysis. Esophagus. Published online February 16, 2021. doi:10.1007/s10388-021-00820-6

12. Carson DA, Bhat S, Hayes TCL, et al. Abnormalities on Electrogastrography in Nausea and Vomiting Syndromes: A Systematic Review, Meta-Analysis, and Comparison to Other Gastric Disorders. Dig Dis Sci. 
Published online May 6, 2021. doi:10.1007/s10620-021-07026-x

13. Varghese $C$, Carson DA, Bhat $S$, et al. Clinical associations of functional dyspepsia with gastric dysrhythmia on electrogastrography: A comprehensive systematic review and metaanalysis. Neurogastroenterol Motil. Published online April 8, 2021:e14151.

14. Carson DA, O'Grady G, Du P, Gharibans AA, Andrews CN. Body surface mapping of the stomach: New directions for clinically evaluating gastric electrical activity. Neurogastroenterol Motil. Published online December 4, 2020:e14048.

15. Verhagen MA, Van Schelven LJ, Samsom M, Smout AJ. Pitfalls in the analysis of electrogastrographic recordings. Gastroenterology. 1999;117(2):453-460.

16. Gharibans AA, Coleman TP, Mousa H, Kunkel DC. Spatial Patterns From High-Resolution Electrogastrography Correlate With Severity of Symptoms in Patients With Functional Dyspepsia and Gastroparesis. Clin Gastroenterol Hepatol. 2019;17(13):2668-2677.

17. Somarajan S, Muszynski ND, Olson JD, et al. The effect of chronic nausea on gastric slow wave spatiotemporal dynamics in children. Neurogastroenterol Motil. Published online November 20, 2020:e14035.

18. O'Grady G, Angeli TR, Du P, et al. Abnormal initiation and conduction of slow-wave activity in gastroparesis, defined by high-resolution electrical mapping. Gastroenterology. 2012;143(3):589-598.e3.

19. Erickson JC, Hayes JA, Bustamante M, et al. Intsy: a low-cost, open-source, wireless multi-channel bioamplifier system. Physiol Meas. 2018;39(3):035008.

20. Gharibans AA, Kim S, Kunkel D, Coleman TP. High-Resolution Electrogastrogram: A Novel, Noninvasive Method for Determining Gastric Slow-Wave Direction and Speed. IEEE Trans Biomed Eng. 2017;64(4):807-815.

21. Gharibans AA, Smarr BL, Kunkel DC, Kriegsfeld LJ, Mousa HM, Coleman TP. Artifact Rejection Methodology Enables Continuous, Noninvasive Measurement of Gastric Myoelectric Activity in Ambulatory Subjects. Sci Rep. 2018;8(1):5019.

22. Abell TL, Camilleri M, Donohoe $\mathrm{K}$, et al. Consensus recommendations for gastric emptying scintigraphy: a joint report of the American Neurogastroenterology and Motility Society and the Society of Nuclear Medicine. J Nucl Med Technol. 2008;36(1):44-54.

23. O'Grady G, Angeli TR, Paskaranandavadivel N, et al. Methods for High-Resolution Electrical Mapping in the Gastrointestinal Tract. IEEE Rev Biomed Eng. 2019;12:287-302.

24. Gharibans AA, Coleman T, Mousa H, Kunkel D. High-Density Multichannel Electrode Array Improves the Accuracy of Cutaneous Electrogastrography Across Subjects With Wide-Ranging BMI: 1235. Am J 
Gastroenterol. 2018;113:S708.

25. McAdams E. Bioelectrodes. Encyclopedia of Medical Devices and Instrumentation. Published online 2006. doi:10.1002/0471732877.emd013

26. Ruenruaysab K, Calder S, Hayes T, O'Grady G, Gharibans A, Du P. Effects of Anatomical Variations on Body Surface Gastric Mapping. Conf Proc IEEE Eng Med Biol Soc. 2020;2020:2388-2391.

27. Rentz AM, Kahrilas P, Stanghellini V, et al. Development and psychometric evaluation of the patient assessment of upper gastrointestinal symptom severity index (PAGI-SYM) in patients with upper gastrointestinal disorders. Qual Life Res. 2004;13(10):1737-1749.

28. De la loge C, Trudeau E, Marquis P, et al. Cross-cultural development and validation of a patient selfadministered questionnaire to assess quality of life in upper gastrointestinal disorders: The PAGIQOL@. Qual Life Res. 2004;13(10):1751-1762.

29. Koch KL, Stern RM. Handbook of Electrogastrography. Oxford University Press; 2004.

30. Yin J, Chen JDZ. Electrogastrography: Methodology, Validation and Applications. Journal of Neurogastroenterology and Motility. 2013;19(1):5-17. doi:10.5056/jnm.2013.19.1.5

31. Du P, O'Grady G, Cheng LK, Pullan AJ. A Multiscale Model of the Electrophysiological Basis of the Human Electrogastrogram. Biophysical Journal. 2010;99(9):2784-2792. doi:10.1016/j.bpj.2010.08.067

32. Bortolotti M. Electrogastrography: a seductive promise, only partially kept. Am J Gastroenterol. 1998;93(10):1791-1794.

33. Calder S, O'Grady G, Cheng LK, Du P. A Simulated Anatomically Accurate Investigation Into the Effects of Biodiversity on Electrogastrography. IEEE Transactions on Biomedical Engineering. 2020;67(3):868-875. doi:10.1109/tbme.2019.2922449

34. Angeli TR, Cheng LK, Du P, et al. Loss of Interstitial Cells of Cajal and Patterns of Gastric Dysrhythmia in Patients With Chronic Unexplained Nausea and Vomiting. Gastroenterology. 2015;149(1):56-66.e5.

35. Bradshaw LA, Cheng LK, Chung E, et al. Diabetic gastroparesis alters the biomagnetic signature of the gastric slow wave. Neurogastroenterol Motil. 2016;28(6):837-848.

36. Kurniawan JF, Tjhia B, Wu VM, et al. An Adhesive-Integrated Stretchable Silver-Silver Chloride Electrode Array for Unobtrusive Monitoring of Gastric Neuromuscular Activity. Advanced Materials Technologies. 2021;6(5):2001229. doi:10.1002/admt.202001229

37. Vijayvargiya P, Jameie-Oskooei S, Camilleri M, Chedid V, Erwin PJ, Murad MH. Association between delayed gastric emptying and upper gastrointestinal symptoms: a systematic review and metaanalysis. Gut. 2019;68(5):804-813. 
38. Tack J, Schol J, Horowitz M. Gastroparesis: A Dead-end Street After All? Gastroenterology. 2021;160(6):1931-1933.

39. Lacy BE, Crowell MD, Cangemi DJ, Lunsford TN, Simren M, Tack J. Diagnostic Evaluation of Gastric Motor and Sensory Disorders. Official journal of the American College of Gastroenterology / ACG. 2021;116(12):2345.

40. Patcharatrakul T, Gonlachanvit S. Technique of functional and motility test: how to perform antroduodenal manometry. J Neurogastroenterol Motil. 2013;19(3):395-404.

41. Camilleri M. Testing the sensitivity hypothesis in practice: tools and methods, assumptions and pitfalls. Gut. 2002;51 Suppl 1:i34-i40.

42. O'Grady G, Du P, Cheng LK, et al. Origin and propagation of human gastric slow-wave activity defined by high-resolution mapping. Am J Physiol Gastrointest Liver Physiol. 2010;299(3):G585-G592.

43. Berry R, Miyagawa T, Paskaranandavadivel $\mathrm{N}$, et al. Functional physiology of the human terminal antrum defined by high-resolution electrical mapping and computational modeling. Am J Physiol Gastrointest Liver Physiol. 2016;311(5):G895-G902.

44. Chan C-HA, Aghababaie Z, Paskaranandavadivel N, Avci R, Cheng LK, Angeli-Gordon TR. Localized gastric distension disrupts slow-wave entrainment leading to temporary ectopic propagation: a highresolution electrical mapping study. Am J Physiol Gastrointest Liver Physiol. 2021;321(6):G656-G667.

45. O'Grady G, Gharibans AA, Du P, Huizinga JD. The gastric conduction system in health and disease: a translational review. Am J Physiol Gastrointest Liver Physiol. 2021;321(5):G527-G542.

46. Bhat S, Varghese C, Carson DA, et al. Electrogastrography Abnormalities in Pediatric Gastroduodenal Disorders: A Systematic Review and Meta-Analysis. J Pediatr Gastroenterol Nutr. Published online March 31, 2021. doi:10.1097/MPG.0000000000003140

47. Berry R, Cheng LK, Du P, et al. Patterns of Abnormal Gastric Pacemaking After Sleeve Gastrectomy Defined by Laparoscopic High-Resolution Electrical Mapping. Obes Surg. 2017;27(8):1929-1937.

48. Wang TH-H, Angeli TR, Beban G, et al. Slow-wave coupling across a gastroduodenal anastomosis as a mechanism for postsurgical gastric dysfunction: evidence for a "gastrointestinal aberrant pathway." Am J Physiol Gastrointest Liver Physiol. 2019;317(2):G141-G146.

49. Tack J, Corsetti M, Camilleri M, et al. Plausibility criteria for putative pathophysiological mechanisms in functional gastrointestinal disorders: a consensus of experts. Gut. 2018;67(8):1425-1433.

50. Hunt JN, Smith JL, Jiang CL. Effect of meal volume and energy density on the gastric emptying of carbohydrates. Gastroenterology. 1985;89(6):1326-1330. 
51. Calder S, Cheng LK, Andrews C. Validation of non-invasive body-surface gastric mapping for detecting electrophysiological biomarkers by simultaneous high-resolution serosal mapping in a porcine .... bioRxiv. Published online 2021. https://www.biorxiv.org/content/10.1101/2021.08.01.454685.abstract

52. O'Grady G, Gharibans A, Calder S, Andrews C. Retrograde slow-wave activation: a missing link in gastric dysfunction? Neurogastroenterol Motil. 2021;33(4):e14112.

53. Calder S, O'Grady G, Cheng LK, Peng Du. A Theoretical Analysis of Electrogastrography (EGG) Signatures Associated With Gastric Dysrhythmias. IEEE Trans Biomed Eng. 2017;64(7):1592-1601.

54. Cheng S, Kupfer K, Dixon M, Shammas S. Optimized sampling design and rationale for verification and validation. Qual Reliab Eng Int. 2019;35(1):483-502.

\section{Figures}




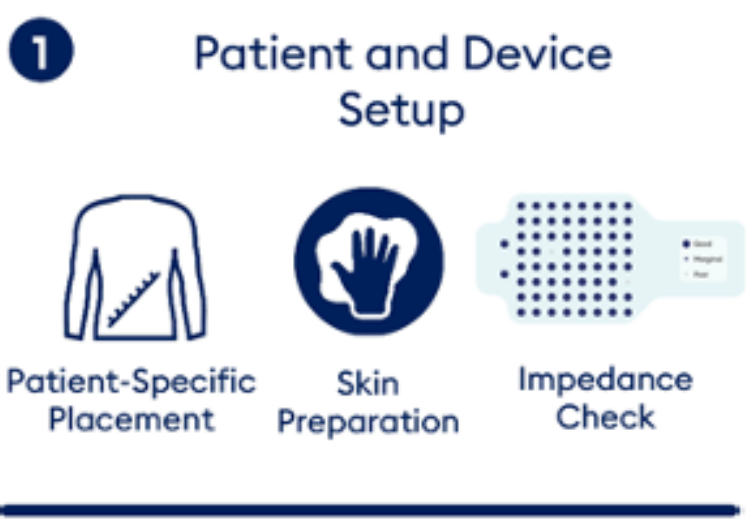

\section{Gastric Data Capture}

High-resolution bioelectrical signal acquisition

continuous
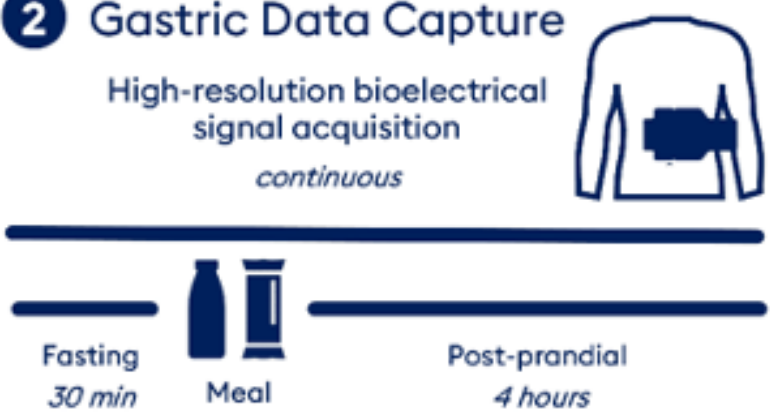

(3) Biomarker Reporting and Visualization

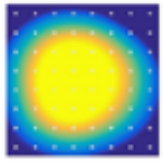

Signal Strength

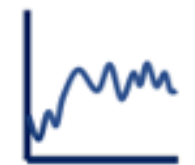

Frequency/ Time

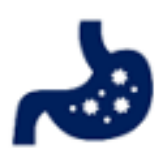

Meal Response

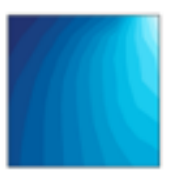

Wave

Propagation

\section{Figure 1}

Body Surface Gastric Mapping (BSGM) workflow. (1) Setup includes placement of the sensor array personalized by measurements between anatomic landmarks, skin preparation with conductive gel, and a signal check using live impedance data displayed on the companion App. (2) Gastric activity is captured in HR continuously throughout the recording period (30 m fasted, standardized meal, up to 4 hours postprandially). (3) A report of gastric activity is generated following automated signal processing and analyses. This includes a heat map used to infer gastric position by spatial distribution of amplitude, traditional gastric biomarkers including frequency and amplitude, along with novel meal response and spatial wave propagation biomarkers. 

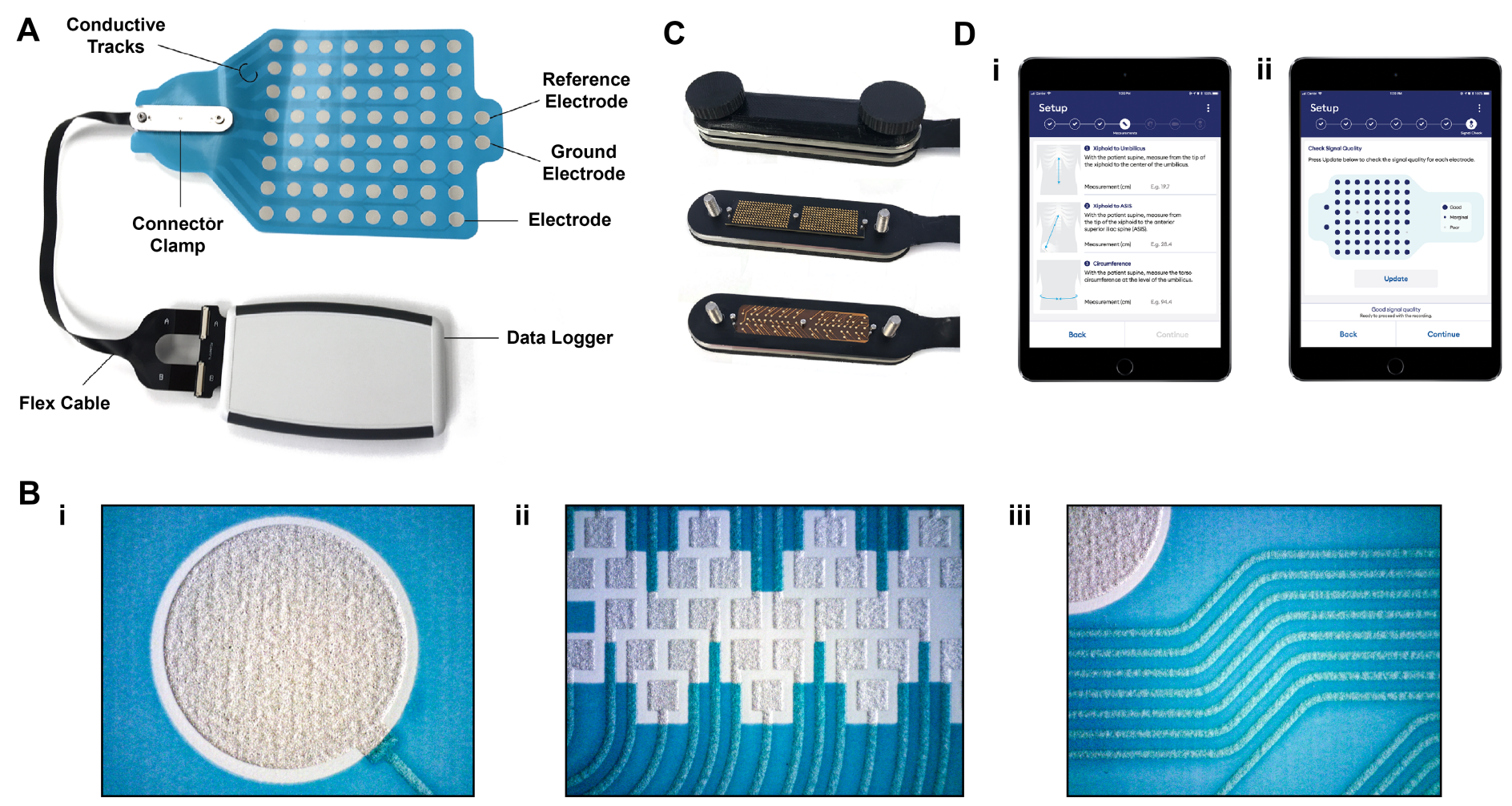

\section{Figure 2}

Components of Body Surface Gastric Mapping (BSGM) system. (A) Assembled BSGM system including sensor array, connector clamp, flexible printed circuit cable, and wearable data logger. (B) Close-up of sensor array mating panel, demonstrating convergence of all 64 conductive tracks. This is opposed with the cable using the connector clamp shown in (C): thumb screws are loosened and the top piece removed to expose the connector piece (middle) which facilitates secure connection between the mating panel and the connector cable (bottom). (D) Companion App used to register test and participant details, customize recording variables, and guide setup of the sensor array and data logger. (D. i) Measurement input interface, where distances between the xiphoid and umbilicus, xiphoid and anterior superior iliac spine (ASIS), and abdominal circumference are recorded to guide personalized array positioning. (D. ii) Signal quality check shown on the App. The size and color of each electrode button represents the impedance measured for each channel. 
A

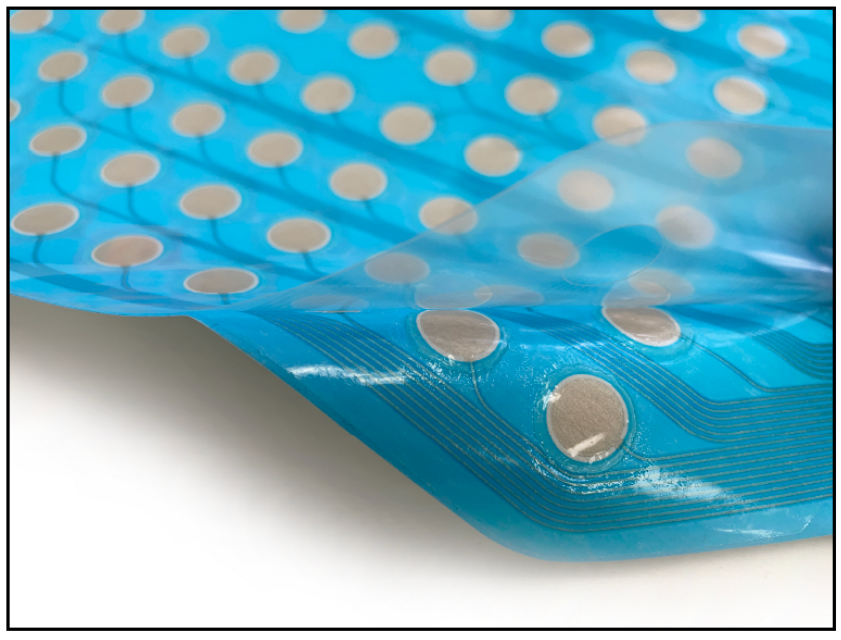

B

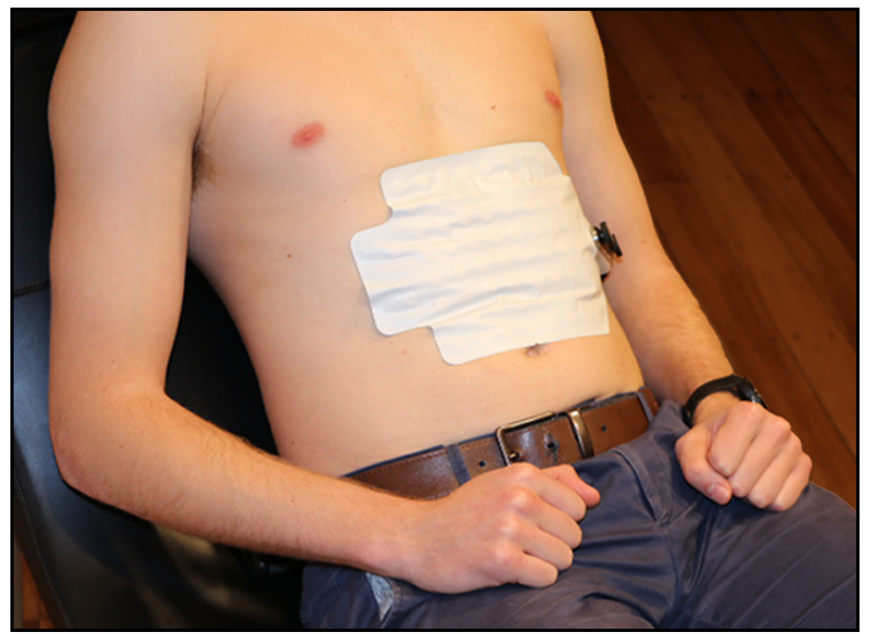

\section{Figure 3}

High-resolution sensor array ( 8 × 8 pre-gelled $\mathrm{Ag} / \mathrm{AgCl}$ electrode grid; $2 \mathrm{~cm}$ spacing; 64 channels; area 225 $\mathrm{cm}^{2}$ ) printed upon a flexible TPU substrate, enabling comfort and optimal electrode contact across contours of the abdominal wall. (A) Backing layer partially peeled off, exposing adhesive layer and hydrogel discs. (B) Sensor array placed on a subject's epigastrium while reclined at 45 degrees.
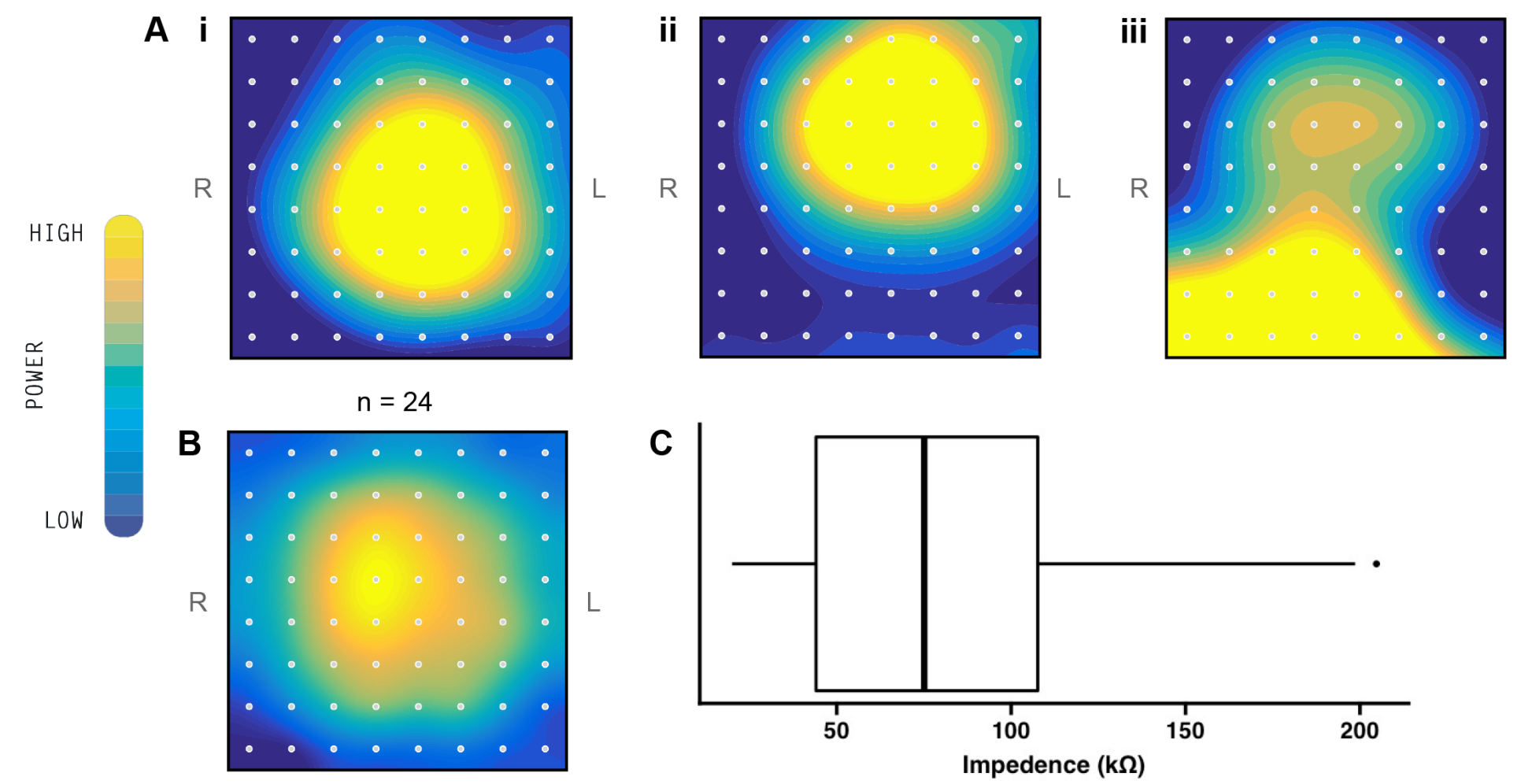

Figure 4 
(A) Spatial heat maps for three participants, demonstrating the estimated signal power in the gastric range over the recording duration. Each electrode is represented by a gray circle, and the subject's left $(L)$ and right $(\mathrm{R})$ are indicated. Approximate stomach location can be inferred by the region of greatest amplitude (yellow). (B) Average heat map for all 24 participants. (C) Box and whisker plot of mean impedance for all 24 subjects.
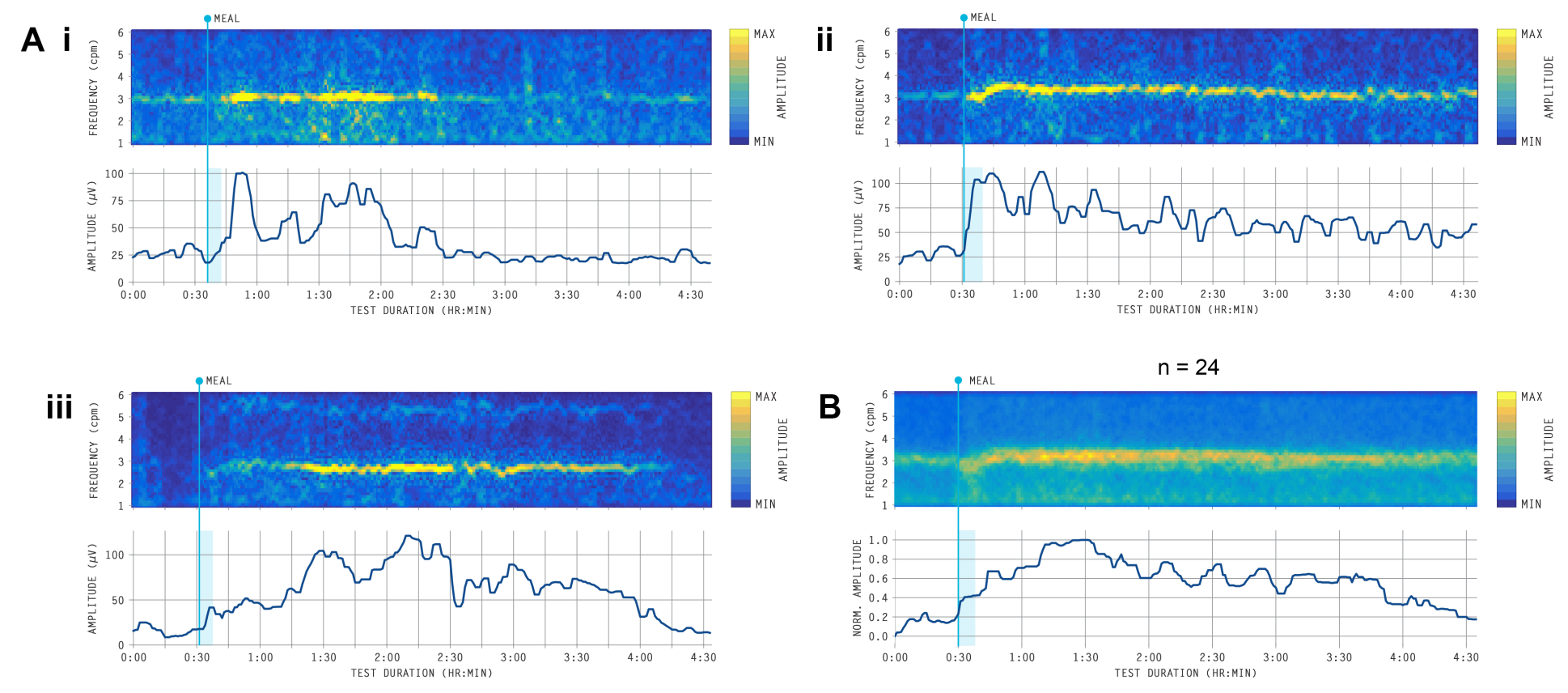

\section{Figure 5}

(A) Frequency-amplitude spectrograms (top) and amplitude over time (bottom) for three subjects demonstrating features of the typical meal response. Start of meal time is marked by the blue line. Variability in the meal response was observed, with near-immediate postprandial increases in amplitude (e.g. (i) and (ii)), or following a lag phase (e.g. (iii)). (B) Average frequency-amplitude spectrogram for all 24 subjects, after normalization for amplitude. Amplitude steadily increased following the meal to reach a plateau at 1 hour postprandially, before gradual return towards baseline (gasting) amplitude by the end of the 4 hour postprandial period. 
A

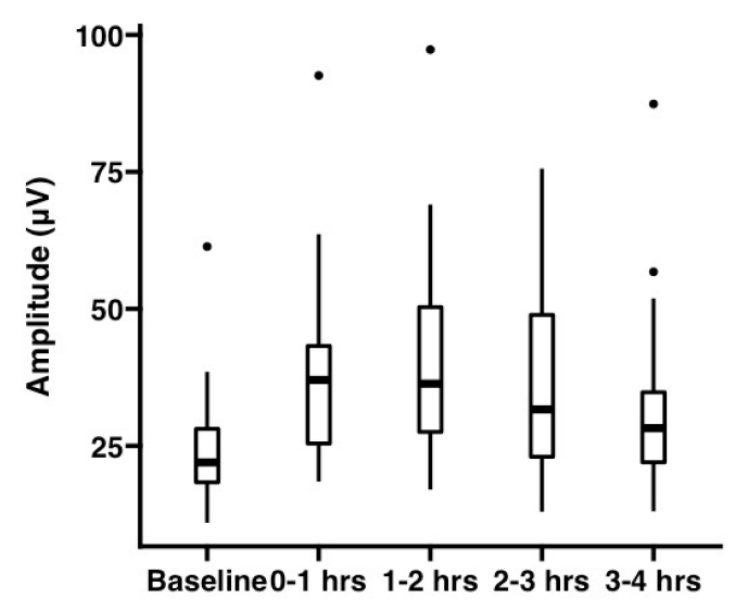

B

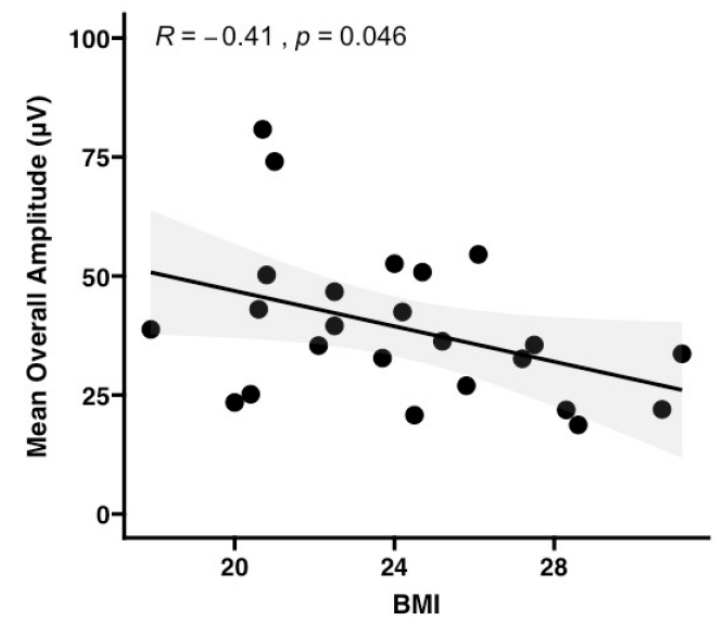

Figure 6

(A) Box and whisker plots of cohort mean amplitude over $1 \mathrm{~h}$ time periods. (B) Scatter plot of mean amplitude and BMI with a linear trend line applied.

A

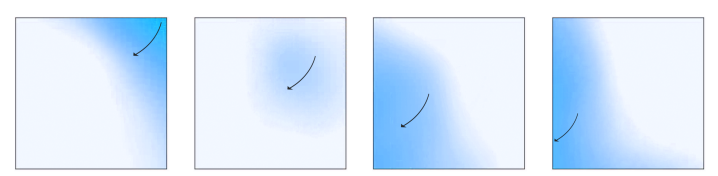

Antegrade (30-45 min postprandial)

B i
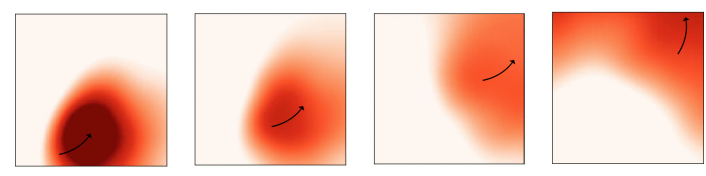

Retrograde (15-30 min postprandial)

ii
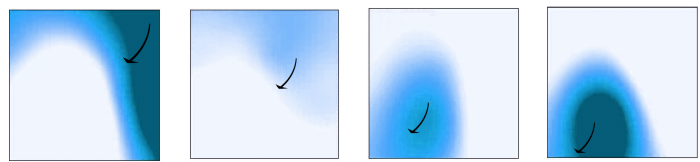

Antegrade (75-90 min postprandial)

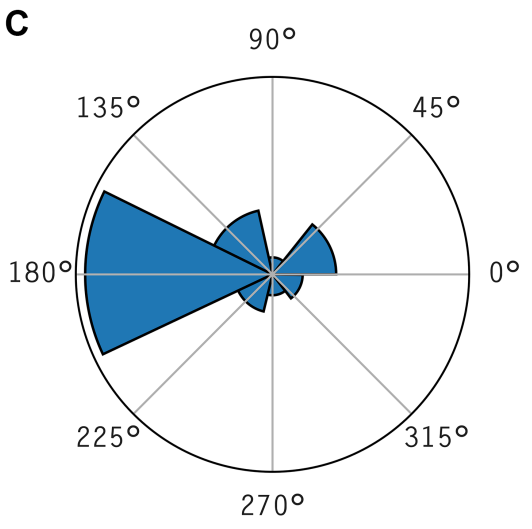

D

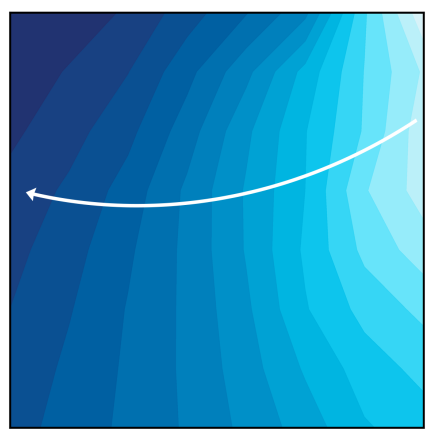

\section{Figure 7}

(A) Representative animations of postprandial periods of subject with antegrade activity; (B) representative animations of postprandial periods of subject with (i) retrograde activity immediately postprandially, which returned to antegrade activity soon after (ii); (C) Average polar histogram of all 24 subjects; (D) Average phase map of all 24 subjects.

\section{Supplementary Files}


This is a list of supplementary files associated with this preprint. Click to download.

- RetrogradeBi.mp4

- AntegradeBii.mp4

- AntegradeAi.mp4

- SupplementaryMethodsandFigures.docx

- FigureS1.png 